\title{
Hipomagnesemia en recién nacidos con encefalopatía hipóxico isquémica en hipotermia corporal total
}

\author{
Hypomagnesemia in newborns with hypoxic ischemic encephalopathy and \\ whole-body hypothermia
}

\author{
Andrea Maccioni Romero ${ }^{\mathrm{a}}$, Patricia Mena Nannig ${ }^{\mathrm{a}, \mathrm{b}}$ \\ aServicio de Neonatología, Complejo Asistencial Dr. Sótero del Río \\ bDepartamento de Neonatología, División de Pediatría, Pontificia Universidad Católica de Chile
}

Recibido: 27 de mayo de 2019; Aceptado: 17 de noviembre de 2019

¿Qué se sabe del tema que trata este estudio?

Recién nacidos con encefalopatía hipóxico-isquémica en tratamiento con hipotermia tienen peor pronóstico si presentan alteraciones metabólicas y la hipomagnesemia es frecuente. El magnesio tiene un rol neuro-protector, existiendo diversas publicaciones que muestran mejoría en resultados neurológicos a corto plazo con el uso de sulfato de magnesio postnatal, pero su uso farmacológico aún no se ha establecido como un estándar de tratamiento.

\section{¿Qué aporta este estudio a lo ya conocido?}

Confirma la alta frecuencia de hipomagnesemia en recién nacidos con encefalopatía hipóxico-isquémica en tratamiento con hipotermia.

La hipomagnesemia debe ser controlada desde las primeras horas de vida y corregida a valores normales neonatales de forma adecuada con aporte de sulfato de magnesio.

\section{Resumen}

En recién nacidos (RN) con encefalopatía hipóxico isquémica (EHI) en hipotermia se describen alteraciones metabólicas que se asocian a pronóstico neurológico. La hipomagnesemia ha sido reportada en la literatura, pero no es medida ni corregida en todos los centros de atención neonatal. Objetivo: Evaluar la frecuencia de hipomagnesemia e hipocalcemia en RN con EHI en tratamiento con hipotermia corporal total y evaluar la respuesta al aporte de sulfato de magnesio. Pacientes y Método: Estudio prospectivo, observacional y descriptivo en RN con EHI sometidos a hipotermia corporal total, hospitalizados entre los años 2016-2017. Se realizó medición seriada en sangre de magnesemia $(\mathrm{Mg})$ y calcemia $(\mathrm{Ca})$. Con $\mathrm{Mg}$ menor o igual de $1,8 \mathrm{mg} / \mathrm{dl}$ se administró suplemento como sulfato de Mg para mantener niveles entre 1,9 y 2,8 mg/dl. Se describió la frecuencia de hipomagnesemia e hipocalcemia y su presentación en el tiempo. Se realizó registro prospectivo de evolución clínica. Se hizo un análisis estadístico descriptivo, con medidas de tendencia central. Resultados: Se incluyeron 16 pacientes. Presentaron hipomagnesemia $13 / 16$ (81,3\%), la que fue precoz (6-36 h de vida), normalizándose con aporte de sulfato de magnesio, requiriendo $2^{\text {a }}$ dosis 4 de ellos. Presentaron hipocalcemia 6/16 (37,5\%). Conclusiones: La hipomagnesemia es frecuente (80\%), similar a lo descrito en la literatura. Dado su importancia fisiológica debe controlarse y corregirse, de igual manera que el calcio.
Palabras clave:

Encefalopatía hipóxica isquémica; hipotermia; hipomagnesemia; hipocalcemia 


\begin{abstract}
In newborns with the diagnosis of hypoxic-ischemic encephalopathy (HIE) treated with hypothermia, metabolic alterations are observed, which are associated with neurological prognosis. Hypomagnesemia has been reported frequently in the literature in these patients, but it is not measured or corrected in all neonatal healthcare centers. Objective: To evaluate the frequency of hypomagnesemia and hypocalcemia in newborns with HIE treated with whole-body hypothermia and to evaluate the response to the magnesium sulfate administration. Patients and Method: Prospective, observational and descriptive study in hospitalized newborns with the diagnosis of HIE and treated with whole-body hypothermia between the years 2016 and 2017. Serial blood measurement of magnesemia $(\mathrm{Mg})$ and calcemia $(\mathrm{Ca})$ was performed. When presenting an $\mathrm{Mg}$ level $\leq 1.8 \mathrm{mg} / \mathrm{dl}$, supplementation with magnesium sulfate was administered to maintain levels between 1.9 and 2.8 $\mathrm{mg} / \mathrm{dl}$. The frecuency of hypomagnesemia, hypocalcemia and clinical evolution was registered. A descriptive statistical analysis was performed, with central tendency measures. Results: Sixteen cases were included, 13 of them presented hypomagnesemia (81.3\%), with early-onset (6-36 hours of life), which was normalized with magnesium sulfate treatment, receiving a second dose 4 patients. Six of 16 patients presented hypocalcemia $(37.5 \%)$. Conclusions: Hypomagnesemia is frequent $(80 \%)$, similar to that described in the literature, and should be controlled and corrected early, given its physiological role, in the same way that calcium is controlled.
\end{abstract}

Keywords:

Hypoxic-Ischemic Encephalopathy; hypothermia; hypomagnesemia; hypocalcemia

\section{Introducción}

La encefalopatía hipóxico isquémica (EHI) es una causa significativa de morbilidad y mortalidad en recién nacidos (RN) de término. En EHI moderada o grado II se describe una mortalidad de un $10 \%$ y secuelas de un 30\%; en EHI severa o grado III $60 \%$ y $100 \%$ respectivamente ${ }^{1,2}$. Su incidencia es de $1 / 1.000$ $\mathrm{RN}$ vivos en países desarrollados y 5-10/1.000 en países en vías de desarrollo 3 .

La hipotermia corporal total moderada $\left(33-34^{\circ} \mathrm{C}\right.$ por $72 \mathrm{~h}$ ) es una de las terapias que ha mostrado efectividad en reducir el daño neurológico en RN con EHI grado II y III al iniciarse dentro de las primeras $6 \mathrm{~h}$ de vida en RN de 36 o más semanas de edad gestacional. Reduce la mortalidad o discapacidad neurológica moderada a severa a los 18-22 meses de edad desde un 62\% en el grupo control a $44 \%$ en el grupo con hipotermia $(\mathrm{p}=0,01)$, la mortalidad desde $37 \%$ a $24 \%(\mathrm{p}=0,08)$ y aumenta la sobrevida libre de discapacidad ${ }^{1,4-6}$. Los mecanismos involucrados en la neuroprotección con el uso de hipotermia son varios, entre ellos, disminución: del metabolismo, del consumo de $\mathrm{O}_{2}$ y producción de $\mathrm{CO}_{2}$, de la pérdida de fosfatos de alta energía, de citotoxicidad, de la producción de especies de oxígeno reactivas y del edema. Además modula la cascada inflamatoria, los cambios en la señalización pro y anti apoptótica y preserva la síntesis de proteínas ${ }^{7}$.

Los pacientes con EHI presentan un compromiso multisistémico, que lleva a variadas alteraciones metabólicas, algunas de los cuales constituyen factores protectores o también de mal pronóstico ${ }^{8}$. La hipomagnesemia se ha descrito en pacientes con EHI e hipotermia en los últimos años ${ }^{9,10}$. El magnesio $(\mathrm{Mg})$ tiene un rol neuroprotector al ser un antagonista no competitivo de receptores $\mathrm{N}$-methyl-D-aspartate (NMDA), los cuales son mediadores de la muerte neuronal secundaria a hipoxia isquemia. Al bloquear los receptores NMDA, disminuye el influjo de calcio $(\mathrm{Ca})$ a la célula, lo que limita el daño mitocondrial y la muerte neuronal ${ }^{10}$.

La medición de magnesio sérico en $\mathrm{RN}$ con $\mathrm{EHI}$ no es una práctica habitual, como lo es la medición de los niveles de calcio. Es poco conocido que recién nacidos sanos y prematuros presentan un aumento de los niveles de $\mathrm{Mg}$ después del nacimiento y que en presencia de hipocalcemia y enfermedad el Mg no se eleva ${ }^{11,12}$. En $\mathrm{RN}$ con EHI tratados con hipotermia se ha reportado hipomagnesemia ${ }^{10}$.

El objetivo de este trabajo es describir la frecuencia de hipomagnesemia en RN con EHI en tratamiento con hipotermia corporal total y la respuesta al suplemento con sulfato de magnesio para normalizar los niveles séricos de $\mathrm{Mg}$.

\section{Pacientes y Método}

Estudio de cohorte prospectivo, observacional y descriptivo. Se aplicó un protocolo de control de magnesemia desde las $6 \mathrm{~h}$ de vida a todos los recién nacidos hospitalizados en el Servicio de Neonatología del Complejo Asistencial Dr. Sótero del Río, con diagnóstico de EHI grado II o III según criterios de Sarnat y Sarnat ${ }^{13}$, $\geq 35$ semanas de gestación, tratados con hipotermia corporal total moderada, con equipo Blanketrol ${ }^{\circledR}$, durante el período de marzo del 2016 a septiembre 
Tabla 1. Control calcemia y magnesemia según protocolo

\begin{tabular}{lccccccc}
\hline & $6 \mathrm{~h}$ & $12 \mathrm{~h}$ & $24-36 \mathrm{~h}$ & $48-60 \mathrm{~h}$ & $72-96 \mathrm{~h}$ & 5 días \\
\hline Magnesio & $\mathrm{x}$ & $\mathrm{x}$ & $\mathrm{x}$ & $\mathrm{x}$ & $\mathrm{x}$ & $\mathrm{x}$ \\
Calcio & & $\mathrm{x}$ & $\mathrm{x}$ & $\mathrm{x}$ & $\mathrm{x}$ & $\mathrm{x}$ \\
\hline
\end{tabular}

Tabla 2. Suplementación con sulfato de magnesio y gluconato de calcio según protocolo

\begin{tabular}{ll}
\hline $\begin{array}{l}\text { Magnesemia mg/dl } \\
1,9-2,8\end{array}$ & Conducta a seguir \\
$1,4-1,8$ & Valores normales administrar endovenoso lento (1 hora) \\
$<1,4$ & Sulfato de magnesio $25 \% 25 \mathrm{mg} / \mathrm{kg}$ diluido, administrar endovenoso lento (1 hora) \\
Calcemia mg/dl & Sulfato de magnesio $25 \% 50 \mathrm{mg} / \mathrm{kg}$ diluido, administrar endovenoso lento (1 hora) \\
$7,5-9,5$ & Conducta a seguir \\
$<7,5$ & Valores normales administrar endovenoso lento (1 hora) \\
& Gluconato de calcio $10 \% 1 \mathrm{ml} / \mathrm{kg}$ diluido, administrar en $1 \mathrm{~h}$ cada $8 \mathrm{~h}$ endovenoso o hasta que la \\
\hline
\end{tabular}

de 2017. No se incluyeron pacientes malformados ni casos con encefalopatía de posible causa metabólica o infecciosa, ni pacientes cuya madre recibió tratamiento con sulfato de magnesio previo al parto. En las tablas 1 y 2 se describe el control y administración de calcio y magnesio utilizado.

Se estableció un nivel de magnesemia entre 1,9 y $2,8 \mathrm{mg} / \mathrm{dl}$ como óptimo. En la literatura los valores considerados como hipomagnesemia varían de una población estudiada a otra. Generalmente se cita 1,7 $\mathrm{mg} / \mathrm{dl}$ como valor límite, pero dado que los niveles de $\mathrm{Mg}$ suben postparto en $\mathrm{RN} \operatorname{sanos}^{11} \mathrm{y}$ que algunos reportes en neonatología y pediatría han considerado 1,8 $\mathrm{mg} / \mathrm{dl}$ como límite inferior, éste fue el nivel de referencia utilizado en el estudio ${ }^{10,14-16}$.

Se realizó un análisis estadístico descriptivo, con medidas de tendencia central.

El estudio fue aprobado por el Comité de Ética e Investigación del Complejo Asistencial Dr. Sótero del Río.

\section{Resultados}

Se reclutaron 16 pacientes cuyas características demográficas y clínicas se describen en la tabla 3. La edad gestacional promedio fue de 39 semanas, con un rango entre 36 y 41 semanas, siendo un 56,2\% de sexo masculino. Fueron adecuados para edad gestacional 14 pacientes $(87,5 \%)$ y $2(12,5 \%)$ pequeños para edad gestacional, con un promedio de peso al nacer de $3.350 \mathrm{~g}$. El ingreso a protocolo de hipotermia fue en promedio a las 3,5 h de vida, con un rango de 0,5 a 7 h. En la tabla
3 se detallan los valores de exceso de base en cordón o punción de paciente durante la primera hora de vida, APGAR y grado de encefalopatía hipóxico isquémica. El 43,8\% (7/16) presentaron EHI grado IIA, un 43,8\% (7/16) presentaron EHI grado IIB y un $12,5 \%(2 / 16)$ EHI grado III.

En cuanto a la evolución neonatal 43,8\% (7/16) de los pacientes estuvieron en ventilación mecánica invasiva, con una mediana de 2,6 días. Presentaron convulsiones un $62,5 \%(10 / 16)$ de los pacientes. Un 50\% (8/16) requirieron drogas vasoactivas (dopamina, dobutamina y/o epinefrina) con un rango de duración de 2 a 6 días y un 18,8\% (3/16) de los pacientes recibieron hidrocortisona en dosis de estrés $\left(50 \mathrm{mg} / \mathrm{m}^{2} /\right.$ día $)$. El aporte enteral se inició entre los 3 y 6 días de vida y el volumen enteral total se alcanzó entre los 7 y 26 días. La alimentación por vía oral se logró entre los 4 y 28 días. La duración de la hospitalización varió entre 10 y 47 días, con una mediana de 16 días.

Se observó hipomagnesemia $\leq 1,8 \mathrm{mg} / \mathrm{dl}$ en $13 \mathrm{de}$ 16 casos $(81,3 \%)$, en su mayoría en las primeras $60 \mathrm{~h}$ de vida y en un caso se presentó entre las 72-96 h. En la tabla 3 se detalla el menor valor de $\mathrm{Mg}$ de cada caso. El valor mínimo de magnesemia fue 1,4 y el máximo 2,8 mg/dl. En todos los casos con hipomagnesemia se administró sulfato de magnesio, persistiendo la hipomagnesemia en 4/13 (30,8\%) pacientes, por lo que se administró una segunda dosis, con lo cual se normalizó el valor de la magnesemia. Presentaron hipocalcemia $6 / 16$ casos $(37,5 \%)$ que requirieron aporte de calcio (gluconato de calcio $10 \% 1 \mathrm{ml} / \mathrm{kg}$ diluido al medio cada $8 \mathrm{~h}$ ev hasta llegar a calcemia $>8 \mathrm{mg} / \mathrm{dl}$, según protocolo). 
Tabla 3. Variables demográficas y clínicas de 16 recién nacidos con Encefalopatía Hipóxico Isquémica

\begin{tabular}{|c|c|c|c|c|c|c|c|c|c|c|}
\hline $\mathrm{N}^{\circ}$ & PN g & EG sem & Sexo & $\mathrm{BE}$ & Apgar 1 y $5^{\prime}$ & Grado EHI & Menor Mg & Menor $\mathrm{Ca}$ & Aporte Mg & Aporte Ca \\
\hline 1 & 3.450 & 39 & $M$ & $-24,2$ & $4-6$ & $\| A$ & 1,64 & 7,7 & + & \\
\hline 2 & 3.645 & 39 & M & -19 & $2-5$ & $\| B$ & 1,6 & 7,9 & + & \\
\hline 3 & 3.280 & 41 & $\mathrm{~F}$ & $-7,1$ & $4-7$ & $\| B$ & 1,57 & 7,7 & + & \\
\hline 4 & 3.435 & 39 & M & -12 & $6-8$ & $\| \mathrm{A}$ & 1,4 & 8 & + & \\
\hline 5 & 3.360 & 40 & $\mathrm{~F}$ & -14 & $3-8$ & $\| \mathrm{A}$ & 1,65 & 7,5 & + & + \\
\hline 6 & 3.055 & 37 & M & -17 & $2-5$ & $\| B$ & 1,67 & 9 & + & \\
\hline 7 & 3.490 & 39 & $\mathrm{~F}$ & $-0,2$ & $3-5$ & $\| \mathrm{A}$ & 1,86 & 7,7 & & \\
\hline 8 & 3.810 & 38 & M & -12 & $4-5$ & $\| \mathrm{A}$ & 1,71 & 8,1 & + & \\
\hline 9 & 2.960 & 38 & $\mathrm{~F}$ & $-7,6$ & $3-5$ & $\| \mathrm{B}$ & 1,46 & 8 & + & \\
\hline 10 & 4.075 & 39 & $\mathrm{~F}$ & -19 & & $\| B$ & 1,78 & 7,8 & + & \\
\hline 11 & 3.385 & 41 & $\mathrm{~F}$ & $-7,5$ & $2-5$ & $\| \mathrm{A}$ & 1,7 & 7,7 & + & \\
\hline 12 & 2.640 & 36 & $\mathrm{M}$ & -26 & $0-3$ & III & 1,85 & 7,2 & & + \\
\hline 13 & 3.265 & 40 & $M$ & -19 & $4-6$ & $\| \mathrm{A}$ & 1,89 & 7,2 & & + \\
\hline 14 & 2.125 & 38 & $\mathrm{~F}$ & -19 & $1-4$ & III & 1,73 & 7,2 & + & + \\
\hline 15 & 2.630 & 36 & $\mathrm{M}$ & -15 & $3-4$ & $\| B$ & 1,41 & 6,3 & + & + \\
\hline 16 & 3.340 & 39 & $\mathrm{~F}$ & -18 & $3-7$ & $\| B$ & 1,4 & 6,7 & + & + \\
\hline
\end{tabular}

PN: peso de nacimiento, EG: edad gestacional, sem: semanas, M: masculino, F: femenino, BE: exceso de base en cordón o de paciente a los 0-60 minutos de vida, EHI: encefalopatía hipóxico isquémica, II: moderada, IIA: sin convulsiones, IIB: con convulsiones, III: severa, Mg: magnesio, Ca: calcio.

\section{Discusión}

En el paciente con EHI, en tratamiento con hipotermia corporal, junto con las alteraciones metabólicas habitualmente descritas (hipo/hiperglicemia, hiponatremia, hipocalcemia) puede presentarse hipomagnesemia. En este estudio confirmamos lo descrito en la literatura sobre la disminución del magnesio sérico y su aumento con aporte suplementario en niveles de 25 a $50 \mathrm{mg} / \mathrm{Kg}$ de sulfato de $\mathrm{Mg}$ al $25 \%$. Su importancia radica en que estas alteraciones metabólicas juegan un rol tanto en pronóstico neurológico como en morbimortalidad $^{8,9,17,18}$.

Los resultados de este estudio coinciden con la literatura en la frecuencia de hipomagnesemia. Se describe una frecuencia de hipomagnesemia $(<1,6-1,85 \mathrm{mg} / \mathrm{dl})$ de un $80-89 \%$ en pacientes con EHI especialmente en hipotermia ${ }^{9,10}$. En caso de EHI sin hipotermia se describe también hipomagnesemia ${ }^{9,23}$, con menores niveles a mayor grado de $\mathrm{EHI}^{12}$. Los niveles de cordón y cercanos al nacimiento están influenciados por la administración de sulfato de $\mathrm{Mg}$ a la madre ${ }^{19}$, pero en este estudio ninguna madre recibe $\mathrm{Mg}$ prenatal.

El magnesio es un catión abundante intracelular, sólo el $1 \%$ se encuentra en el espacio extracelular. Es esencial para muchas reacciones metabólicas, cofactor de reacciones enzimáticas, tiene un rol en la síntesis de proteínas y ácidos nucleicos, en la integridad citoesquelética/mitocondrial y modula el transporte transcelular (bombas, transportadores y canales). El rol regulatorio del magnesio en la calcemia es conocido, a través de la estimulación o inhibición de la hormona paratiroidea (HPT). En caso de hipomagnesemia, disminuye la calcemia porque la hipomagnesemia suprime la liberación de HPT y además induce la resistencia de los órganos a la acción de la hormona ${ }^{20}$. El calcio forma parte de los mecanismos de daño neuronal al entrar a la célula vía receptores NMDA, desencadenando el daño mitocondrial y muerte neuronal. El magnesio tiene un rol neuro-protector al ser un antagonista no competitivo de receptores NMDA bloqueando el ingreso de calcio a la célula ${ }^{10,11,21}$. En diferentes patologías con procesos inflamatorios y a nivel experimental se ha mostrado que la liberación de citoquinas y otras moléculas oxidantes podrían ser causantes de hipomagnesemia ${ }^{22}$. La sustancia $\mathrm{P}$ parece tener un rol importante en mediar el daño asociado a hipomagnesemia ${ }^{22}$.

El uso de suplemento de sulfato de magnesio no se ha establecido como una práctica clínica en pacientes con EHI durante la hipotermia, pero parece fundamental mantener la homeostasis metabólica ${ }^{9,20,23}$. Su uso en dosis farmacológicas en RN con EHI en hipotermia como neuro-protector no ha mostrado aumento de eventos adversos, su efectividad a corto plazo ha mostrado resultados favorables y a mediano/largo plazo se encuentra en estudio ${ }^{21}$. Las dosis empleadas en este estudio son mucho menores que las administradas en estudios de suplemento de Mg en pacientes con EHI en hipotermia, cuya práctica no se ha generalizado ${ }^{5}$. En una revisión sistemática que utiliza suplemento de sul- 
fato de magnesio (125-250 mg/kg en 30-60 min) en el primer día de vida, en RN en hipotermia, describieron menor riesgo de anormalidad en el examen clínico y neuroimágenes en la hospitalización ${ }^{24}$.

Pacientes que han presentado hipomagnesemia, se ha reportado mayor daño cerebral evaluado por resonancia nuclear magnética cerebral ${ }^{9}$. El estudio multicéntrico aleatorizado (MAG COOL STUDY) comparó hipotermia asociado a sulfato de magnesio $(250 \mathrm{mg} / \mathrm{kg}$ cada $24 \mathrm{~h}$ por 3 dosis, administrada en 30 min e iniciada dentro de las primeras $6 \mathrm{~h}$ de vida) v/s hipotermia asociado a placebo (suero fisiológico) en recién nacidos $\geq 35$ semanas. No hubo diferencias en mortalidad entre ambos grupos, ni eventos adversos severos con el aporte de $\mathrm{Mg}^{25}$. Un meta-análisis con 5 estudios aleatorizados-controlados en $\mathrm{RN}$ de término concluía que existe una mejoría en resultados desfavorables a corto plazo definidos como aquellos que se producen en $<$ 18 meses y presentan sobrevida con anormalidades en cualquiera de los siguientes: examen del neurodesarrollo, neuroimagen, estudios neurofisiológicos. No hubo diferencias en mortalidad, convulsiones e hipotensión $^{26}$. Estudios en animales han mostrado que la asociación entre hipotermia y sulfato de magnesio es más efectiva en disminuir muerte neuronal que cada uno por separado y que éste último se asocia con aumento de la perfusión cerebral y disminución de la resistencia vascular periférica ${ }^{20}$.

En cuanto a los efectos adversos que podrían ser secundarios al uso de sulfato de magnesio como hipotensión que requiera drogas vasoactivas (DVA) y depresión respiratoria que requiera ventilación mecánica invasiva (VMI), los estudios no muestran aumento de éstos $^{9,24,25}$. Los valores encontrados en este estudio de uso de VMI $(43,8 \%)$ y DVA (50\%) son bastantes similares a los reportados en la literatura (VMI 55-100\%, DVA $35-87,1 \%)^{23,25,27}$.

En RN de término con EHI en hipotermia aún no hay consenso en suplementar con sulfato de $\mathrm{Mg}$ de forma postnatal ${ }^{9,20,24-27}$. Se requiere un estudio aleatorizado, controlado, de mayor tamaño, para evaluar mejor el efecto neuro-protector a largo plazo y tener consenso en la dosis y tiempo de administración ${ }^{27}$.

El uso de sulfato de magnesio prenatal se ha asociado a menor riesgo de parálisis cerebral en prematuros $\leq 33$ semanas y se ha establecido como una práctica clínica $^{19,28-30}$.

La hipomagnesemia en EHI e hipotermia se ha descrito en la frecuencia antes mencionada, incluso con uso de nutrición parenteral precoz ${ }^{10}$. No hay consenso en el inicio precoz o tardío de la nutrición parenteral en pacientes con EHI en tratamiento con hipotermia ${ }^{31}$ y se cuestiona el uso de nutrición parenteral precoz en el recién nacido en situación crítica, por los eventos adversos asociados al aporte de aminoácidos en estos $\operatorname{casos}^{32}$.
Dentro de las limitaciones de este estudio encontramos el bajo número de pacientes reclutados durante el período de tiempo del estudio, lo que se puede explicar porque la EHI no es una patología frecuente. Además se administró magnesio en cantidad dietaria $(25-50 \mathrm{mg} / \mathrm{kg} / \mathrm{dosis})$ más que farmacológica, lo que debiera ajustarse, con lo que disminuiría la persistencia de hipomagnesemia. No se realizó un estudio controlado aleatorizado para observar los cambios de calcemia con la administración de magnesio, lo cual es difícil por el tamaño muestral. A pesar del bajo número de casos, permitió establecer que gran parte de los niños con EHI en hipotermia evolucionan con hipomagnesemia que requiere corrección, lo cual es compatible con lo descrito en la literatura. La fortaleza del estudio es advertir sobre la necesidad de controlar la magnesemia periódicamente, desde las $6 \mathrm{~h}$ de vida hasta el término del calentamiento y corregir los niveles bajos en los RN con EHI sometidos a hipotermia. A futuro sería importante evaluar cómo el manejo de la magnesemia influye en la necesidad de suplementar calcio y en el daño neuronal en esta población durante su seguimiento.

\section{Conclusiones}

Hipomagnesemia e hipocalcemia son frecuentes en $\mathrm{RN}$ con EHI en hipotermia corporal total. El control de los niveles séricos de magnesio permite establecer frecuencia de hipomagnesemia en pacientes con EHI en hipotermia y su corrección a niveles fisiológicos. En la literatura existen estudios que muestran mejoría en resultados neurológicos a corto plazo con el uso de sulfato de magnesio en $\mathrm{RN}$ con $\mathrm{EHI}$ en hipotermia, pero faltan estudios sobre el efecto a largo plazo, por lo que aún no hay consenso en el uso de aportes farmacológicos en este tipo de pacientes. A pesar de lo último, existe una alerta sobre la importancia del magnesio, de su medición y corrección en estos pacientes.

\section{Responsabilidades Éticas}

Protección de personas y animales: Los autores declaran que los procedimientos seguidos se conformaron a las normas éticas del comité de experimentación humana responsable y de acuerdo con la Asociación Médica Mundial y la Declaración de Helsinki.

Confidencialidad de los datos: Los autores declaran que han seguido los protocolos de su centro de trabajo sobre la publicación de datos de pacientes.

Derecho a la privacidad y consentimiento informado: Este estudio ha sido aprobado por el Comité de Ética de Investigación correspondiente, quien de acuerdo 
a las características del estudio ha eximido el uso del Consentimiento Informado.

\section{Conflicto de intereses}

Los autores declaran no tener conflicto de intereses.

\section{Agradecimientos}

Agradecemos a todo el equipo de salud del Servicio de Neonatología del Complejo Asistencial Dr. Sótero del Río que colaboraron e hicieron posible la realización de este proyecto.

\section{Referencias}

1. Shankaran S, Laptook A, Ehrenkranz $\mathrm{R}$, et al. Whole-Body Hypothermia for Neonates with Hypoxic-Ischemic Encephalopaty. N Engl J Med 2005;353:1574-84.

2. Gluckman P, Wyatt J, Azzopardi S, et al. Selective head cooling with mild systemic hypothermia after neonatal encephalopathy: multicentre randomised trial. Lancet 2005;365:663-70.

3. McGuire W. Perinatal asphyxia. Clinical Evidence 2007;11:320

4. Azzopardi D, Strohm B, Marlow N, et al. Effects of Hypothermia for Perinatal Asphyxia on Childhood Outcomes. N Engl J Med 2014;371:140-9.

5. Grupo de Trabajo Hipotermia Terapéutica, Comité de Estudios FetoNeonatales (CEFEN). Recomendación para el tratamiento con hipotermia en recién nacidos con encefalopatía hipóxico-isquémica. Resumen ejecutivo. Arch Argent Pediatr 2017;115:300-3.

6. Shankaran S, Pappas A, McDonald S, et al. Childhood Outcomes after Hypothermia for Neonatal Encephalopathy. N Engl J Med 2012;366:2085-92.

7. Hassell K, Ezzati M, Alonso-Alconada D, Hausenloy D, Robertson N. New horizons for newborn brain protection: enhancing endogenous neuroprotection. Arch Dis Child Fetal Neonatal Ed 2015;100:F54152.

8. Basu S, Kaiser J, Guffey D, Minard C, Guillet R, Gunn A. Hypoglycaemia and hyperglycaemia are associated with unfavourable outcome in infants with hypoxic ischaemic encephalopathy: a post hoc analysis of the CoolCap Study. Arch Dis Child Fetal Neonatal Ed 2016;101:F149-55.

9. Chakkarapani E, Chau V, Poskitt K, et al. Low plasma magnesium is associated with impaired brain metabolism in neonates with hypoxic-ischaemic encephalopathy. Acta Paediatr. 2016;105:1067-73

10. Tocco N, Hodge A, Jones A, Wispe J, Valentine C. Neonatal Therapeutic Hypothermia-Associated Hypomagnesemia During Parenteral Nutrition Therapy. Nutr Clin Pract 2014;29:246-8.

11. David L, Anast CS. Calcium metabolism in newborn infants. The interrelationship of parathyroid function and calcium, magnesium, and phosphorus metabolism in normal, "sick," and hypocalcemic newborns. J Clin Invest. 1974;54:287-96.

12. Ilves P, Kiisk M, Soopold T, Talvik T. Serum total magnesium and ionized calcium concentrations in asphyxiated term newborn infants with hypoxicischaemic encephalopathy. Acta Paediatr 2000;89:680-5.

13. Sarnat H, Sarnat M. Neonatal Encephalopathy Following Fetal Distress. Arch Neurol. 1976;33:696-705.

14. Verive MJ, Irazuzta J, Steinhart CM, Orlowski JP, Jaimovich DG. Evaluating the frequency rate of hypomagnesemia in critically ill pediatric patients by using multiple regression analysis and a computer-based neural network. Crit Care Med. 2000; 28(10):3534-9.

15. Guerrero-Romero F, Rodríguez-Morán M, Hernández-Ronquillo G, et al. Low Serum Magnesium Levels and Its Association with High Blood Pressure in Children. J Pediatr. 2016;168:93-8.

16. Ghasemi A, Syedmoradi L, Zahediasl S, Azizi F. Pediatric reference values for serum magnesium levels in Iranian subjects. Scandinavian Journal of Clinical \& Laboratory Investigation 2010;70:41520.

17. Galinsky R, Bennet L, Groenendaal F, et al. Magnesium Is Not Consistently Neuroprotective for Perinatal HypoxiaIschemia in Term-Equivalent Models in Preclinical Studies: A Systematic Review. Dev Neurosci 2014;36:73-82.

18. Chouthai NS, Sobczak H, Khan R, Subramanian D, Raman S, Rao R. Hyperglycemia is associated with poor outcome in newborn infants undergoing therapeutic hypothermia for hypoxic ischemic encephalopathy. J. Neonatal Perinatal Med. 2015;8:125-31.

19. Monterrosa-Castro A, Romero-Pérez I, Caraballo-Olave E. Sulfato de magnesio y protección neuronal prenatal. Rev Chil Obstet Ginecol 2013; 78: 201-8.

20. Ayuk J, Gittoes Neil JL. Contemporary view of the clinical relevance of magnesium homeostasis. Ann Clin Biochem 2014, 51:179-88.

21. Lingam I, Robertson NJ. Magnesium as a neuroprotective agent: a review of its use in the fetus, term infant with Neonatal Encephalopaty, and adult stroke patient. Dev Neurosci 2018; 40:1-12.

22. Weglicki WB. Hypomagnesemia and Inflammation: Clinical and Basic Aspects. Annu. Rev. Nutr. 2012; 32:55-71.
23. Ichiba H, Yokoi T, Tamai H, Ueda T, Kim TJ, Yamano T. Neurodevelopmental outcome of infants with birth asphyxia treated with magnesium sulfate. Ped Internat 2006;48: 70-5.

24. Tagin M, Shah PS, Lee K-S. Magnesium for newborns with hypoxic-ischemic encephalopathy: a systematic review and meta-analysis. J Perinatol 2013;33:663669.

25. Rahman S, Canpolat F, Oncel M, et al. Multicenter Randomized Controlled Trial of Therapeutic Hypothermia Plus Magnesium Sulfate Versus Therapeutic Hypothermia Plus Placebo in the Management of Term and Nearterm Infants with Hypoxic Ischemic Encephalopathy (Tha Mag Cool Study): A Pilot Study. J Clin Neonatol 2015;4:15863.

26. Nair J, Kumar VHS. Review Current and Emerging Therapies in the Management of Hypoxic Ischemic Encephalopathy in Neonates. Children (Basel). 2018;5:99.

27. Bhat MA, Charoo BA, Bhat JI, Ahmad SM, Ali SW, Mufti MU. Magnesium sulfate in severe perinatal asphyxia: a randomized, placebo-controlled trial. Pediatrics 2009;123:e764-9.

28. Hirtz DG, Weiner SJ, Bulas D, et al. Antenatal Magnesium and Cerebral Palsy in Preterm Infants. J Pediatr. 2015;167:834-9.

29. Paulsen ME, Dietz RM. Antenatal magnesium for preterm delivery reduces risk of cerebral palsy among surviving very preterm infants. Acta Paediatr. 2018;107:175.

30. Wolf HT, Huusom L, Weber T, et al; EPICE Research Group. Use of magnesium sulfate before 32 weeks of gestation: a European populationbased cohort study. BMJ Open. 2017;27;7:e013952.

31. Battersby C, Longford N, Patel M, et al. Study protocol: optimizing newborn nutrition during and after neonatal therapeutic hypothermia in the United Kingdom: observational study of routinely collected data using propensity matching. BMJ Open 2018;8:e026739.

32. van Puffelen E, Vanhorebeek I, Joosten KFM, Wouters PJ, Van den Berghe G, Verbruggen SCAT. Early versus late parenteral nutrition in critically ill, term neonates: preplanned secondary subgroup analysis of the PEPaNic multicenter randomized controlled trial. Lancet Child Adolesc Health. 2018;2(7):505-15. 